\title{
Control Automation to Reduce Costs of Control
}

\author{
Rob Christiaanse ${ }^{1}$ and Joris Hulstijn ${ }^{2}$ \\ ${ }^{1}$ VU University and EFCO Solutions, Amsterdam \\ ${ }^{2}$ Delft University of Technology, Delft \\ r.christiaanse@efco-solutions.nl, j.hulstijn@tudelft.nl
}

\begin{abstract}
Much compliance effort concerns adherence to contracts. Parties to a contract need to make sure the other party fulfills the contract. To this end they may require additional controls in the business process. Controls have costs. In this paper we argue that fully automated controls help to lower control costs, because (i) the can help prevent misstatements (compliance by design) or (ii) they increase the quality of audit evidence and thereby reduce the audit risk and additional audit fees. The line of reasoning is illustrated by a case study of the implementation of automated controls on the procurement process for public transport services for the elderly and disabled. The case study suggests open issues, which can be linked to concepts from Normative Multi Agent Systems.
\end{abstract}

Keywords: compliance monitoring, auditing, evidence.

\section{Introduction}

Business reality consists of contractual arrangements between actors, like seller and buyer. A contract is a statement of intent to regulate behaviour. In this sense, “... most organizations are simply legal fictions which serve as a nexus for a set of contracting relationships among individuals" [17] (p.310). Within a larger rulegoverned setting a contract provides for a comprehensive set of both constitutive and regulative rules [23]. Constitutive rules introduce abstract classifications of facts and entities and by describing the (legal) consequences of actions, whereas regulative norms provide conditional obligations with an associated sanction or reward [4]. Such provision can be fruitfully studied using Normative Multiagent Systems [7].

Businesses put more and more effort into collecting evidence to demonstrate compliance with contracts. Much of the (corporate) governance debate concentrates on the key question: "What constitutes an efficient control system?" [24, 27, 29]. One way to deal with increasing administrative burden, i.e., the cost of control, is to use information technology in a clever fashion. Generally, information systems may help (i) to collect and possibly even analyse evidence, and (ii) to facilitate the organization to be 'in control' by preventing undesired behaviour. This may be called 'compliance by design'[22]. The term was initially used in the context of business process management and is related to conformance checking [21]. Conformance checking assumes there is a reference model (de jure model) with process constraints against which the evidence of process behaviour (de facto model) can be verified. But is is no 
specified how to derive the reference model, e.g. from legal sources, technical standards or best practices. Also, given a de facto model, how the raw evidence needs to be interpreted and mapped onto the de jure model is left unspecified. Issues due to transaction costs, auditing roles and responsibilities and the meaning of evidence are not sufficiently addressed. We therefore prefer to use the term 'compliance by design' in a broader sense, referring to an integrated design of organizational, procedural and technical measures, to make sure the organization is evidently compliant.

In this paper we analyse the problem of assurance provision within a fully automated environment, focusing on the strength of evidence and the roles of management, auditors and stakeholders in setting up a control system to minimize the compliance control costs. Our research question is twofold:

(1) How can organizations ensure and prove to others that they are keeping the contract, while minimizing the costs of control?

Our approach will be to analyse a real world case study. The case study concerns the set-up of automated control measures for ensuring compliance of the monthly invoice with the contract, regulating public transport services for the elderly and disabled.

To analyse the case study we will use techniques from the field of Normative Multi Agent Systems (NMAS) [4, 7]. We believe NMAS concepts bring out observations, which may bridge the gap between abstract norms and systems specifications.

The remainder of the paper is structured as follows. In Section 2 we discuss the various aspects of control and the role of collecting strong evidence. In Section 3 we describe the case study, illustrating the difficulties of a highly regulated application domain. In Section 4 we provide some open issues for research, indicating how Normative Multi-Agents Systems could be used to analyse these issues.

\section{Control, Business Compliance and Assurance}

So what are governance and control? Governance is related to delegation. The owner of a firm must delegate tasks to its members. Delegating tasks raises specific control problems for the principal, in particular the problem of private information [19]. Private information problems can be of two types, namely moral hazard (hidden action) and adverse selection (hidden knowledge). A third type of problem concerns non-verifiability. Non-verifiability of information occurs when a principal and an agent share information ex-post which cannot be observed by a third party, who may also have an interest in the outcomes, for example a regulator.

These control problems are generally countered by additional control measures, like supervision, procedures, verification, or automated application controls, etc. Controls come with a cost. The cost of control depends on two factors: (1) the incremental monetary cost of the control instrument chosen, including opportunity costs and (2) the cost of negative side effects caused by or related to the controls [20]. In particular, there are four types of problems: (2.1) behavioural displacement (alter behaviour to avoid the controls; (2.2) gamesmanship (exploit the rules to individual or organizational advantage, e.g. fully use subsidies even when not needed 
(2.3) operating delays (additional controls like supervision or signing off slow down or disrupt primary processes); and (2.4) negative attitudes (controls may highlight extrinsic motivation, e.g. targets, rather than intrinsic motivation, which has a negative effect on motivation of employees).

How can we measure the costs of controls? In the case of controls being added to contracts, one way to make sense of the costs is to use Transaction Cost Economics (TCE) [29]. In TCE costs are defined as "The ex ante costs of searching, drafting, negotiating and safeguarding an agreement and, more especially, the ex post costs of maladaptation and adjustment that arise when contract execution is misaligned as a result of gaps, errors, omissions, and unanticipated disturbances" [26]. A rational actor will prefer the governance structure with the lowest transaction costs $[11,29]$. Optimal control is achieved, when the expected control losses are smaller than the cost of implementing more controls [20, 24]. From a management perspective there are several strategies to deal with control risks: (1) activity elimination, (2) centralization, (3) risk-sharing, (4) automation and (5) mitigation. Options (1), (2) and (3) are strategic issues; usually these are given. Therefore we will focus on (4) automation and (5) mitigation.

A mayor source of administrative burden is due to errors and mistakes in the transaction data, and in general, lack of information quality. Traditionally these problems are dealt with by the mitigation strategy, reducing errors by additional human checks. Such action controls, like workflow rules, direct supervision, separation of duties, procedures, etc., are rather costly. Another way to deal with the effects of inaccuracy, inconsistency and lack of motivation is to use information technology in a clever fashion. After all, software is never dishonest or disloyal [20]. Built-in controls provide reasonable assurance that performed activities meet process specifications and contracts are kept against acceptable control costs.

The private information problem of agency theory is in practise dealt with by auditors. Auditors are independent experts, monitoring the firm on behalf of stakeholders of the firm. For decision purposes, stakeholders need reliable financial information about the status of an organisation and its performance [12, 18]. Traditionally auditors are responsible for providing reasonable assurance that (financial) information is free from material misstatements [16, 18]. In this respect materiality reflects "... the magnitude of an omission or misstatement of (financial) information that, inferred in the light of surrounding circumstances, makes it probable that the judgment of a reasonable person relying on this (financial) information would have been changed or influenced by the omission or misstatement" [16]. In general, auditors use the audit risk model in planning the audit process: “... the audit risk concerns the risk that the auditor may unknowingly fail to appropriately modify that his or her opinion on (financial) information that are materially misstated" [18].

\section{(2) Audit Risk $=$ Inherent Risk $*$ Control Risk $*$ Detection Risk}

The audit risk model should be thought of as an aid in understanding how various factors affect the amount of substantive testing required. Inherent risk is defined as the a priori likelihood that a management assertion may be misstated, so before considering the possible effect of (internal) controls. Control risk is defined as the 
likelihood that (internal) controls will not prevent or detect a misstatement. Detection risk refers to the risk that an auditor will not detect a material misstatement, and consequently fail to appropriately modify his or her opinion. Detection risks concern failures of substantive testing and the appropriateness of audit evidence, including the strength or persuasiveness of audit evidence.

In general, there are six ways of obtaining audit evidence: (1) Inspection, (2) Confirmation, (3) Observation, (4) Re-performance, (5) Analytical evidence an (6) Client inquiry [18]. Inspection can be subdivided into inspection of tangible assets and inspection of documents and records. There exist a hierarchy of evidence reliability. Types (1), (2) an (4) are considered the best forms, while types (3), (5) and (6) are considered good or weak, depending on the sources (human or automated), expectations, procedures and planning of the client inquiry. Types (1), (2) and (4) are also the most time consuming for the auditor and therefore the most expensive for the client. So there is a trade-off between the quality of evidence and the costs of control.

How can we conceptualize automated controls? Following Clark and Wilson [10] we distinguish the notions of external consistency and internal consistency of the data produced by a system. Suppose we have a well-managed computer system. Its specifications have been verified to be correct, and the system itself has been tested and behaves according to its specifications. That means that when we enter data into the system that is valid, valid data will ensue (internal consistency). However, even in such a near-perfect system there is nothing to ensure external consistency: correspondence with reality. In general, external consistency can only be ensured by a combination of organizational measures (segregation of duties), procedural measures (e.g. maintenance, supervision) and physical measures (e.g. gates, use of IDs). These are called indispensable measures.

Built-in automated controls may have two effects. Clearly, built-in controls help to prevent material misstatements so they reduce the internal control risk. Built-in controls may also mean that audit evidence will be stronger, because of the availability of reliable transaction data. If this is the case, the detection risk will become lower: the probability that the auditor would miss a possible material misstatement is reduced. Therefore, for both these reasons, in principle fewer substantive tests are needed. We summarize this expectation as follows:

(3) Control automation reduces the costs of control, by increasing both

a. the probability of preventing misstatements, and

b. by aiding the auditor improve the audit by strengthening the audit evidence.

In the following section we will explore this double expectation in practice.

\section{The Case of Procurement for Public Transport Services}

We expect that control automation will enhance the control effectiveness and quality of evidence. We investigate this claim by analysis of a real world case. 


\subsection{Case Description}

The case concerns the public procurement process for care-related public trans- port services. In the Netherlands municipalities have the responsibility to procure public transport services, facilitating in particular the elderly, people with functional disabilities and people with mental health problems. As you can imagine this is a highly regulated sector. Relevant provisions are found for instance in European Directive 2004/18/EC about public transport contracts, ensuring the principle of equal treatment, the principle of proportionality and the principle of transparency.

We focus on one particular contract: TaxiBus (TED 2010/S 31-044711 dated $13 / 02 / 2010)$. The case is exemplary for a large class of care-related services. The contract involves the following participants.

The contracting authority is called SRE, procuring the transport services and paying the invoices. SRE represents fifteen municipalities in the Eindhoven region, in the role of stakeholders. SRE is assisted by an external auditor.

The transport service provider (TSP) provides care-related 'taxi bus' services on a demand basis. It receives a monthly fee from SRE as well as individual contributions from (some) passengers. TSP keeps a record of all trips being requested, executed and cancelled, including data about patients and other travellers.

\subsection{Contract}

The contract contains provisions about safety, competence of the drivers, and quality. Other requirements concern the contract area, tariff rules based on the number of travel zones, and the types of clients (residents; elderly; companions). In addition to a fee from SRE, passengers must also pay a contribution. Pensioners pay a lower contribution. The contract contains performance provisions about delays or maximum driving time. When TSP does not meet these criteria a penalty must be paid.

1. The carrier receives a fee based on the number of transportation zones covered in a trip, based on a rate per area. No additional embarkation zone is covered.

a. The carrier charges the fee per area. The carrier charges the net amount of travelled trips, excluding received contributions;

b. All amounts are exclusive of VAT.

c. The carrier may not charge SRE an additional fee, for:

- a medical supervisor (see TED 2010/S 31-044711 par. 1.6.4)

- a companion (see TED 2010/S 31-044711 par 1.6.4); in this case the contribution is collected by the driver;

- children up to four years old;

- guide-dogs or other assistance dogs, recognizable as such.

The carrier may charge trips in addition to the contribution, in case the passenger and his companions are family;

d. The following trips are not reimbursable:

- $\quad$ transport of animals, goods etc;

- trips that do not meet the registration requirements, as set out in TED 2010/S 31-044711 par. 1.17.1 (except GPS-coordinates ); 
- cancelled trips;

- no-show rides.

2. Passenger rate per zone. A passenger pays a contribution based on the number of transportation zones, plus an embarkation zone.

Every month SRE receives an invoice. How can SRE establish the accuracy and legitimacy of the invoice? By contract, the invoice is accompanied by an Excel data file, containing the grounds for billing: the registration of the trips being ordered, executed, and cancelled. For example, patient IDs, scheduled and actual departure and arrival times, locations and so on. The contract contains a data protocol, specifying which data elements the Excel file should contain and in which order.

3. The carrier maintains tamper-resistant automated records of the number of requested and conducted trips, organized in runs based on the route planning software in the central office and on the trip registration device in each vehicle. This means that refused, not implemented or no-show rides are recorded.

4. The carrier shall provide the client with a monthly Excel file, detailed as specified in 5, containing all requested and actually implemented customer trips. This means that refused, not implemented or no-show rides are included.

5. For each one-way trip, at least the following data is registered (selection): unique trip identification number; identification code of any subcontractor who performs the trip; transit pass number (customer identification number), if any, by means of which user and residential municipality can be identified;

municipality where the customer resides;

customer type(WMO customer, elderly customer or regular public transport); agreed departure time;

agreed arrival time;

actual departure time;

actual arrival time;

travel duration in minutes;

departure address;

destination address;

departure and destination public transportation zone;

length of the trip through some specific areas (excluding pick-up zone);

length of the trip mileage;

number of zones to be charged to contracting authority

number of persons to be transported;

type accompanying person (medical supervisor, social companion, nonpaying children, family taxi, etc.);

travellers contribution (including any companions' contribution);

required equipment and aids (wheelchairs, scooters, guide dogs, etc.);

status of the ride (out, refused, not implemented, no-show, implemeted);

This trip administration forms the ground for billing, to be provided to both SRE and the auditor, so that the correctness and completeness of the invoice can be ascertained. 
The Excel file is however being provided by the transport service provider. So the contract stipulates the need for assurance, obtained from an external auditor.

6. "Each year the service provider is required, no later than three months after the end of a calendar year, to present an audit opinion asserting the correctness of the invoices, the delivered data and the management information. This audit opinion is accompanied with a report of findings concerning the comprehensiveness of the accounting information system. The auditing fees are paid by the service provider" (TED 2010/S 31-044711, p 32);

\subsection{Negotiation about Controls}

SRE management has to decide what controls are requested to prevent misstatements in the monthly invoices. However, these controls have to be paid for, by tax payers' money. There is a trade-off between the effectiveness and costs of control. This results in a dialogue between the municipality in the role of stakeholder, and SRE management in the role of contracting authority. Note that such dialogues are common in audit dialogues about the design of a system of controls [9].

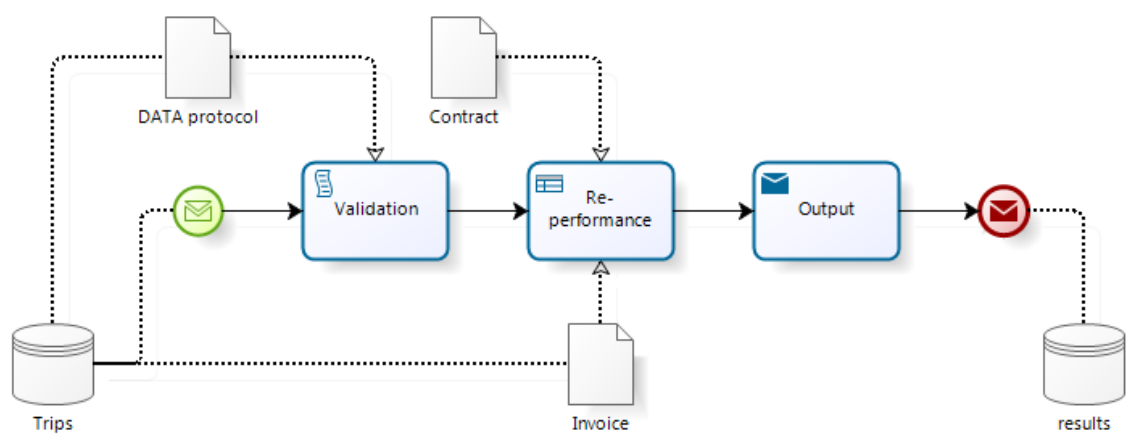

Fig. 1. General overview of establishing accuracy of the monthly invoice

SRE argues that, if TSP is in control of its billing process, the invoice and the data provided in Excel will be accurate. The stakeholder might ask: "How can you be sure?" SRE will respond that the contract obliges TSP "to present an audit opinion asserting the correctness of the invoices, the delivered data and the management information." Now the stakeholder might argue: "Do we not pay too much, because we pay in advance, and when an error is detected by TSP's auditor, it will not lead to any correction of the invoiced amounts, for the detected error will remain within the error threshold?". After some pondering: "No, it seems to me that the invoices are simply illegitimate in case the ground for billing is not correctly established for each trip". . SRE has foreseen this line of reasoning and states: "That is why we receive on a monthly basis an Excel file containing all requested and actually implemented trips.

${ }^{1}$ Law on audit of counties and municipalities, 2003. 
Plus we have a data protocol in which data definitions are defined". The stakeholder responds positively: "So, you are able to verify the grounds for billing using the Excel file accompanying the invoice?" SRE answers positively but claims they do not have the resources to manually check all trips (50.000 per month). The solution for the control problem is therefore full automation, i.e., audit evidence type (4).

\subsection{Control System Specification}

Figure 1 contains a possible architecture for establishing accuracy of the monthly invoice on the basis of an independent evidence file about the trips being conducted. Here we will give semi-formal characterisation of the requirements. The validation procedure is written in PHP. For representing contracts there are better options, such as FCL [14]. Here, we merely want to characterize the kinds of reasoning about the evidence. We will use Predicate Logic notation.

Suppose a contract is a set of clauses $C=\left\{C_{1}, \ldots, C_{\mathrm{n}}\right\}$, where each clause may contain free variables referring to trips, passengers, and other data types. We have a set $E$ of facts about trips. Facts are instantiated and do not contain free variables. Invoice amount $x$ is based on price function ' $f($.$) ' over trips. \varepsilon$ is an acceptable margin of error (materiality). Now we can formulate the control requirements as follows:

$$
\begin{aligned}
& E \mathrm{l}=\forall t\left(C_{1}(t) \wedge \ldots \wedge C_{\mathrm{n}}(t)\right) \\
& \text { Let } y=\Sigma t f(t), \text { for all } t \text { such that } E \mathrm{l}=C_{1}(t) \wedge \ldots \wedge C_{\mathrm{n}}(t) \\
& \text { Invoice } x \text { is accurate when } y-\varepsilon<x<y+\varepsilon .
\end{aligned}
$$

legitimacy

recalculation

accuracy

Concerning evidence $E$, two major issues must be addressed: internal integrity and external integrity [10]. The internal integrity of data refers to conformance of the data to the data protocol. The external integrity depends on the authorization level of the recorded data, and on the presence of segregation of duties and other input controls during generation of the evidence $E$ (indispensable control measures) [2]. Evidence about these internal controls is represented in an audit assertion $I C$, obtained once a year from the external auditor. We suppose the auditor uses a norm $N$ about adequacy of internal controls in a billing process. If needed, the auditor can also verify registered trips against the system's planning module; data about implemented trips is obtained from the taxi drivers' mileage administration; refused and no-show trips can be obtained from passenger day schedules.

However external integrity of the data can also (partly) be established on the basis of the data itself. After all, taken together, the data must form a set of well-formed transactions. This can be verified by so called reconciliation relations, based on accounting theory [25]. Both syntactic constraints and reconciliation relations are specified in a testing procedure $M$.

In our experience, there are at least four stages in a validation procedure $M$. Stage 0 validates whether the evidence file contains the attributes defined in the data protocol. Stage 1 validates the syntax. Stage 2 validates whether the content of an attribute (if defined) conforms to the value constraints (semantics). Stage 3 validates relation constraints, in particular whether two or more attributes (if defined) taken together form a well-formed transaction. 
So, reliability of evidence $E$ is established by comparing evidence of the internal controls $I C$ used in generating $E$ to a norm $N$ (external auditor), and by automatically validating $E$ on the basis of checks and recalculation specified in $M$.

$$
\begin{aligned}
& \text { assert(auditor, } I C \text { meets } N \text { ) } \\
& \text { validate }(E, M)
\end{aligned}
$$

internal controls meet norm $N$ automated validation test $M$

This will work, when $M$ is sound, i.e., when validation test procedure $M$ is strong enough to imply the constraints in the contract.

$$
\text { if validate }(E, M) \text {, then } E \mathrm{l}=\forall t\left(C_{1}(t) \wedge \ldots \wedge C_{\mathrm{n}}(t)\right) \text { soundness }
$$

It is important that all stakeholders can verify soundness of $M$. To facilitate this, the mapping constraints are written in PHP and the validation code is made public.

\section{Organizational Roles in Automated Audit Facilities}

Control and auditing is in essence about setting, following and verifying norms. In the case study we have seen that actors are negotiating about the controls to be included in a contract. Here we are interested in the assurance question: who gets to decide what 'counts as' evidence? Who is verifying that evidence? Who will pay for such assurance services? Such a negotiation suggests a game theoretic setting, because actors reason about what they should do in response to what the other party is likely to do, under condition of a set of controls. In this section we will use ideas from qualitative game theory expressed in Normative Multi-agent Systems [5, 7], to generalize the case study and analyse the roles in assurance provisioning.

Normative multiagent systems are systems composed of autonomous agents with a normative system, i.e. a set of norms (obligations and conventions), to regulate behaviour of the agents. Here we will only use NMAS terminology as a conceptual tool to make an analysis of the stakeholders and their relationships. The particular version we are inspired by [7], makes use of input-output logic, but that is totally irrelevant to the point of the paper. Obviously, there are much better formalisms to express contractual clauses in logic, such as versions of RuleML [13], or to translate contracts into constraints on business processes, such as Formal Contract Logic (FCL) [14] based on defeasible logic [1]. However, here we are not interested in the logical properties or the representation question; the point is agenthood: who decides?

Consider an abstract transaction as depicted in Figure 2. It shows various embedded legal or professional jurisdictions, depicted as spheres. The kernel is made up by the contractual obligations of buyer $b$ and service provider $s$. Both parties can hire an auditor, $a b$ and $a s$ respectively, also regulated by contracts. In particular, $a b$ provides assurance over service delivery, based on evidence provided by $s$. Auditor $a s$ provides an audit assertion about the internal control system of $s$, so indirectly about the way the evidence is being generated. Moreover, $a b$ may take a mediating role in contract negotiations, providing advice about the evidence requirements. Note that auditors have shared professional standards and values about what counts as proper evidence. There are also domain specific provisions (e.g. about health care and public transport) and all of this is set within national or international contract law. 


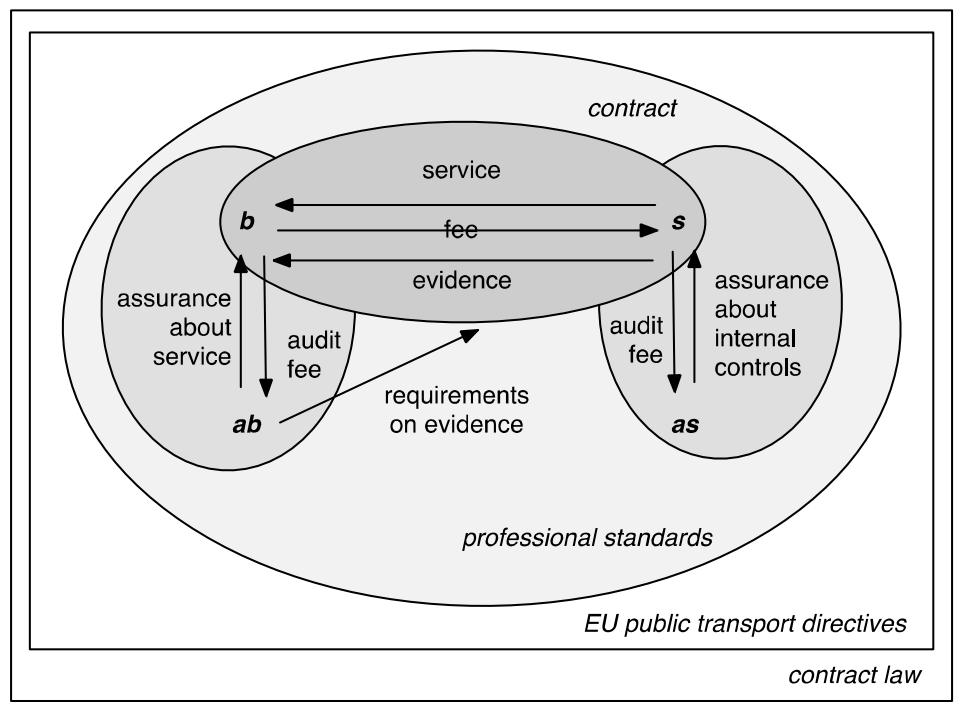

Fig. 2. Legal setting of contract negotiations

As is clear from the picture, we need constitutive rules, based on the distinction between constitutive and regulative rules popularized by Searle [23]. Roughly, constitutive rules help to shape social reality, just like the rules of chess constitute the game of chess. Regulative rules attempt to alter the behaviour of agents within the normative system, by qualifying certain behaviour as violations and making it subject to sanctions. Constitutive rules are generally of the form: "such and such an $X$ counts as $Y$ in context $S$ " where $X$ is any object, fact or event and $Y$ is a label that qualifies $X$ as being of a certain category, often a social or institutional fact. In our case, validation procedure $M$ counts as an acceptable test for an accurate and legitimate invoice. The context $S$ is interpreted as a set of embedded institutional jurisdictions, as in Figure 2. $S$ is common knowledge .For an elaborate analysis of counts-as see [15].

In our simplified NMAS approach, agents are just sets of sets of production rules, with a preference order $<a$. Rules are of the form $p 1, \ldots, p$ n $\rightarrow q$, where all $p i$ and $q$ are literals: facts or negations of facts. When $n=0$, we just have a fact, written $\rightarrow q$. Rules are clustered in sets: belief rules $\mathrm{B} a$ and goal rules $\mathrm{G} a$. To calculate the effect of reasoning with these sets of rules, we need a consequence relation. This is provided by taking the fixed point, where rules are applied in an order specified by a preference relation $<a$. Decisions are consistent sets of outcomes of the consequence relation.

Now we move to the representation of an obligation. Following Boella and van der Torre [7] obligations are analysed in terms of goals of the addressee of the norm, agent $a$, and of the institution or normative agent $\boldsymbol{n}$. The idea is that an obligation is a goal of the normative agent $\boldsymbol{n}$, according to the slogan: "Your wish is my command".

There are six clauses to the analysis [3]. The first clause states that an obligation to achieve or maintain $p$ is the goal of the normative agent to achieve or maintain $p$. The 
second and third clauses deal with violations. Obligations only become visible through defining under which conditions they are not met. The third clause says that in principle, the agent desires no violations. The fourth and fifth clauses deal with sanctions. Normative agent $\mathbf{n}$ prefers not to count behaviour as a violation, and does not want to apply sanctions unless there is a violation; otherwise sanctions would be arbitrary. Finally, the agent really doesn't like the sanction, or it would have no deterring effect.

Definition (Obligation): Agent $a$ is obliged towards normative agent $\mathbf{n}$ to decide to do $x$, in case of condition $Y$, against sanction s, in context of institution $S$, written $\mathrm{S} \mathrm{l}=\operatorname{Obl}(a, \boldsymbol{n}, x, s, Y)$, if and only if:

(1) $Y \rightarrow x \in \mathrm{G} a$

(2) $Y \cup\{\neg x\} \rightarrow \mathrm{V}(\neg x, a) \in \mathrm{Gn}$

(3) $\rightarrow \neg \mathrm{V}(\neg x, a) \in \mathrm{Gn}$

(4) $Y \cup\{\mathrm{V}(\neg x, a)\} \rightarrow s \in \mathrm{Gn}$

(5) $\rightarrow \neg s \in \mathrm{Gn}$

(6) $\rightarrow \neg s \in \mathrm{G} a$ agent $a$ prefers to oblige

$\boldsymbol{n}$ prefers to signal violations, if needed

$\boldsymbol{n}$ prefers not to recognize a violation

$\boldsymbol{n}$ prefers to sanction, if needed

$\boldsymbol{n}$ prefers not to sanction a arbitrarily agent $a$ prefers no sanction

Now lets suppose an agent has a private incentive to violate the obligation, for example, to behave opportunistically. Such an incentive can be represented by an additional clause, specifying that (apparently) violations are preferable.

(7) $\rightarrow \neg x \in \mathrm{G} a$

Now we make several observations. First, this scheme is only going to work, if agent $a$ prefers to comply, i.e. (7) $<a$ (1), or else if the deterring effect of sanctions is larger than the gain of a violation, so (7) $<a(6)$. The normative agent must also make tradeoffs concerning efforts. In the context of such trade-offs, detecting violations should have priority: (3) $<\boldsymbol{n}$ (2). Similarly, in the trade-off about applying sanctions, the normative agent should prefer to apply them when necessary: (5) $<\boldsymbol{n}$ (4).

Given that some of these priorities are set, clause (2) is crucial: it specifies violation conditions. However, it could be the case that $\neg x$ cannot be directly observed. Which agent is authorized to specify what counts as a violation? That is essentially a constitutive question. Boella and van der Torre make the following simplifying move: constitutive rules are modelled by the beliefs of the normative agent $\boldsymbol{n}$. The normative agent further determines what further detection conditions $\mathrm{Z}$ count as sufficient evidence of a violation, i.e. $\neg x$.

(2') $Y \cup\{\neg x\} \rightarrow \mathrm{V}(\neg x, a) \in \mathrm{B} \boldsymbol{n}$

(8) $Z \rightarrow \neg x \in \mathrm{B} n$

In a preference order for a rational agent, belief-rules rules outrank goal-rules, to avoid wishful thinking [8]. By putting clause (2') into the beliefs, the normative agent must detect a violation when the conditions are met. Clause (8) provides further detection conditions $Z$. A separate clause is logically not necessary, but in practice non-compliance can show in many different ways (see example below). 


\subsection{Automated Control}

Now we move back to analyse the case study. Buyer $b$ has an obligation to pay for delivery, with a sanction of discontinuation of the services. Service provider s has an obligation to deliver upon payment, with a sanction of a fine.

$$
\begin{aligned}
& \mathrm{Obl}(b, \boldsymbol{n}, \text { pay, stop deliver, }\{\text { deliver }\}) \\
& \mathrm{Obl}(s, \boldsymbol{n} \text {, deliver, fine, }\{\text { pay }\})
\end{aligned}
$$

Now we focus on clause (2') and clause (8). In terms of the case study, the buyer (SRE) has paid in advance on a monthly basis, but needs to verify delivery.

$$
\{\text { pay }\} \cup\{\neg \text { deliver }\} \rightarrow \mathrm{V}(\neg \text { deliver, } s) \in \mathrm{B} \boldsymbol{n}
$$

First, what evidence counts as non-delivery? These three conditions have been discussed in the case. In the context of contract TED 2010/S 31-044711, the following conditions count as evidence of non-delivery: no qualified audit opinion has been provided about the internal controls $I C$, evidence $E$ has not been validated against mapping $M$, or invoice $x$ differs more than an error threshold from the recalculation.

$$
\begin{aligned}
& \{\neg \exists \text { as (assert }(\text { as, IC meets } N))\} \rightarrow \neg \text { deliver } \in \mathrm{B} \boldsymbol{n}, \\
& \{\neg \text { validate }(E, M)\} \rightarrow \neg \text { deliver } \in \mathrm{B} \boldsymbol{n}, \\
& \left\{\Sigma t f(t)+\varepsilon<x \text {, for } t \text { such that } E \mathrm{l}=C_{1}(t) \wedge \ldots \wedge C_{\mathrm{n}}(t)\right\} \rightarrow \neg \text { deliver } \in \mathrm{B} \boldsymbol{n}
\end{aligned}
$$

Second, which actor plays the role of the normative system $\boldsymbol{n}$ ? We have a number of choices in modelling the situation.

Choice A. The normative system $\boldsymbol{n}$ corresponds to the legal authority, which maintains contract law, in our case, the Dutch legal system, with additional provision from EU directives. That means that formally, obligations of buyer and seller are directed towards a legal abstraction; the fact that they deal with each other can be expressed as part of the contents of the contract and violation conditions $Y$.

Choice B. The normative system corresponds to the other contractual party. That means that both formally and in fact obligations are directed towards each other. It also means that parties themselves recognize violations and apply sanctions.

In the case study, the contractual parties do in fact recognize violations themselves, on the basis of what is considered reliable evidence set by an external auditor, and also the contractual parties themselves are allowed to apply sanctions. Therefore we choose option A. Alternatively, we can distinguish different kinds of $\boldsymbol{n}$, performing different tasks. For instance, we can distinguish a detector agent, performing clause (2) - in the case the buyer's auditor - and a sanctioning agent - in the case the opposite contractual party. For similar ideas of separating the roles of agents see [6]. As we have seen, the contract itself stipulates the use of external evidence of an auditor. The auditor is professionally bound by the norms of the profession and by the general legal setting of Dutch contract law and European Directives. Each of these bounds can again be modelled as an obligation, with corresponding violation clauses (2), (2') and (8) which now stipulate evidence of meta-violation. 


\section{Conclusions}

What is considered an efficient control system? How can we reduce the costs of control? In this paper we have explored the possibilities of automated controls.

The main proposition, that we generated based on agency theory and audit theory, is that automated controls can reduce the costs of control, for two reasons. First, automated controls prevent misstatements and limit the internal control risk. Second, automated controls increase the quality of evidence, and thereby decrease the detection risk. Given a fixed audit risk, the auditor therefore has to perform much less substantive testing. Another benefit is that assurance can be provided when needed.

The role of the auditor is to set standards for evidence (data protocol) and to set up automated validation and verification techniques. An auditor (can be another one) must also verify the irreplaceable controls (internal control, procedures and reliability of the computer system), in order to guarantee external consistency. The main work in verifying accuracy and legitimacy is done by verifying the well-formedness of transactions through reconciliation and recalculation. So in general internal consistency can help to bootstrap external consistency, given data interdependencies and a little circumstantial evidence about reliability of the computer system

The ideas expressed above have been exemplified by a detailed case study about automated controls for verifying compliance to a contract concerning public transport services for the elderly and disabled. This constitutes a heavily regulated domain. The case shows that it is possible to use a monthly data file, provided by the service provider, as evidence of accuracy and legitimacy of the monthly invoice. The data file can be verified automatically. The data is so much interdependent that manipulation will show up in the verification process, unless the provider would deliberately use audit software to manipulate the data. In addition, the software and the billing process which generate the data file are audited once a year.

In the example case, validation is done on a monthly basis, in batch mode. However, the same kind of validation could in principle also be done per row on a dynamic basis. This is a form of continuous monitoring or continuous assurance [28]. Recalculation requires totals, but in principle also recalculation could be done dynamically for any total (per day, per week, per month) as the process develops.

Parties negotiate about the required controls, because controls come at a cost. The whole setting is therefore fruitfully analysed using techniques borrowed from Normative MultiAgent Systems. Crucial is the distinction between regulative rules and constitutive rules, which help to specify what counts as evidence of a violation. Summarizing we can say that the NMAS perspective has forced us to be explicit about (i) who is authorized to set standards of evidence (i.e. the auditor), and (ii) how this evidence provisioning is regulated (i.e., by the data protocol in the contract, and the automated verification procedure) The analysis suggests a nested set of NMASs, regulating the contract at the core, but also the contracts of the auditors involved, embedded in various legal and professional jurisdictions. 


\section{References}

1. Antoniou, G., et al.: Representation results for defeasible logic. ACM Transactions on Computational Logic 2(2), 255-287 (2001)

2. Blokdijk, J.H.: Tests of control in the audit risk model: effective? Efficient? International Journal of Auditing 8, 185-194 (2004)

3. Boella, G., Lesmo, L.: A game theoretic approach to norms. Cognitive Science Quarterly 2(3-4), 492-512 (2002)

4. Boella, G., van der Torre, L.: Regulative and Constitutive Norms in Normative Multiagent Systems. In: Procs. of 9th International Conference on the Principles of Knowledge Representation and Reasoning (2004)

5. Boella, G., van der Torre, L.: Contracts as Legal Institutions in Organizations of Autonomous Agents. In: Proceedings of the Third International Joint Conference on Autonomous, Agents and Multi Agent Systems, AAMAS 2004 (2004)

6. Boella, G., Hulstijn, J., van der Torre, L.: Virtual Organizations as Normative Multiagent Systems. In: Proceedings of the 38th Annual Hawaii International Conference on System Sciences, HICSS 2005, p. 192 (2005)

7. Boella, G., van der Torre, L.: A game theoretic approach to contracts in multiagent systems. IEEE Transactions on Systems, Man and CyBernetics - Part C 36(1), 68-79 (2006)

8. Broersen, J., et al.: Goal Generation in the BOID Architecture. Cognitive Science Quarterly 2(3-4), 431-450 (2002)

9. Burgemeestre, B., Hulstijn, J., Tan, Y.-H.: Value-based Argumentation for Justifying Compliance. Artificial Intelligence and Law 19(2-3), 149-186 (2011)

10. Clark, D.D., Wilson, D.R.: A Comparison of Commercial and Military Computer Security Policies. In: IEEE Symposium on Security and Privacy (1987)

11. Dekker, H.C.: Control of Inter-Organizational Relationships: Evidence on Appropriation Concerns and Cordnation Requirements. Accounting, Organisations and Society 29, 27-49 (2004)

12. FASB, Statement of Financial Accounting Concepts No. 2: Qualitative Characteristics of Accounting Information, Financial Accounting Standards Board (1980)

13. Governatori, G., Rotolo, A.: Modelling Contracts Using RuleML. In: Gordon, T. (ed.) Legal Knowledge and Information Systems (Jurix 2004), pp. 141-150. IOS Press, Amsterdam (2004)

14. Governatori, G., Rotolo, A.: Norm Compliance in Business Process Modeling. In: Dean, M., Hall, J., Rotolo, A., Tabet, S. (eds.) RuleML 2010. LNCS, vol. 6403, pp. 194-209. Springer, Heidelberg (2010)

15. Grossi, D., Dignum, F.P.M., Royakkers, L.M.M., Meyer, J.-J.C.: Collective Obligations and Agents: Who Gets the Blame? In: Lomuscio, A., Nute, D. (eds.) DEON 2004. LNCS (LNAI), vol. 3065, pp. 129-145. Springer, Heidelberg (2004)

16. Houston, R.W., Peters, M.F., Pratt, J.H.: The Audit Risk Model, Business Risk and AuditPlanning Decisions. The Accounting Review 74(3), 281-298 (1999)

17. Jensen, M.C., Meckling, W.H.: Theory of the Firm: Managerial Behavior, Agency Costs and Ownership Structure. Journal of Financial Economics 3(4), 305-360 (1979)

18. Knechel, W., Salterio, S., Ballou, B.: Auditing: Assurance and Risk, 3rd edn. Thomson Learning, Cincinatti (2007)

19. Laffont, J.-J., Martimort, D.: The Theory of Incentives: the Principal-Agent Model. Princeton University Press (2002) 
20. Merchant, K.A.: Modern Management Control Systems, text \& cases. Prentice Hall, New Jersey (1998)

21. Rozinat, A., van der Aalst, W.M.P.: Conformance checking of processes based on monitoring real behavior. Information Systems 33(1), 64-95 (2008)

22. Sadiq, S., Governatori, G.: The journey to business process compliance. In: Cardoso, J., van der Aalst, W.M.P. (eds.) Handbook of Research on Business Process Management, pp. 426-454. IGI Global (2009)

23. Searle, J.R.: The Construction of Social Reality. The Free Press (1995)

24. Speklé, R.F.: Explaining management control structure variety: a transaction cost economics perspective. Accounting, Organizations and Society 26(4-5), 419-441 (2001)

25. Starreveld, R.W., de Mare, B., Joels, E.: Bestuurlijke Informatieverzorging, vol. 1. Samsom, Alphen aan den Rijn (1994) (in Dutch)

26. Swedberg, R.: Economic versus sociological approaches to organization theory. In: Tsoukas, H., Knudsen, C. (eds.) Oxford Handbook of Organization Theory, MetaTheoretical Perspectives. Oxford University Press, Oxford (2003)

27. Tirole, J.: Corporate Governance. Econometrica 69(1), 1-35 (2001)

28. Vasarhelyi, M.A., Alles, M., Kogan, A.: Principles of analytic monitoring for continuous assurance. Journal of Emerging Technologies in Accounting 1(1), 1-21 (2004)

29. Williamson, O.E.: Transaction Cost Economics: The governance of contractual relations. Journal of Law and Economics 22, 3-61 (1979) 